
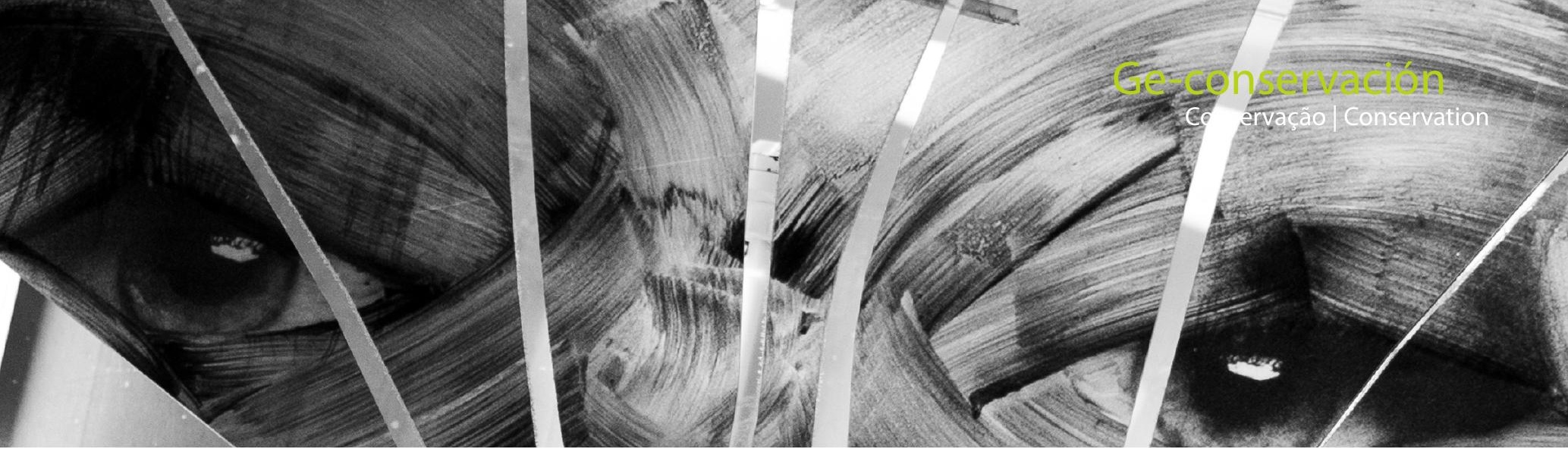

\title{
Galerías, casas de subasta y Urban Art: evolución y paralelismos en las prácticas de arte independiente
}

\author{
Rita Lucía Amor García
}

Resumen: Con la expansión de las prácticas de arte público independiente y su reconocimiento como arte accesible y cercano al público, sus formas se introdujeron y expandieron en entornos tradicionales como las galerías. Con ello, el mercado del arte tomó una importante posición al aceptar dentro de su repertorio obras vinculadas al grafiti y al Street Art (arte urbano). Este arte de origen independiente encontrado actualmente en sectores institucionalizados es lo que se conoce internacionalmente como Urban Art.

A efectos históricos, el nacimiento, evolución y traspaso de fronteras entre las prácticas en espacio público y su inclusión en la galería, son hechos cercanos entre sí y relativamente recientes. Aun así, mucho ha ocurrido desde la celebración de las primeras exposiciones en los años 70 del siglo XX hasta la actualidad. Este artículo hará un recorrido de ese periodo, presentando cómo ha repercutido la inclusión de obras de escritores de grafiti y artistas urbanos dentro del mercado del arte y con ello, en su conservación.

Palabras clave: subasta, arte urbano, grafiti, Urban Art, galerías, arte público independiente, mercado, arte

\section{Art Galleries, auction houses and urban art: evolution and parallelisms in independent art practices}

Abstract: With the expansion of independent public art practices and their recognition as accessible and acceptable to the public, their forms were introduced and expanded into traditional environments such as galleries and auction houses. Consequently, specialists, gallerists and auctioneers recognised the commercial potential of these works, and accepted graffiti and street art within the contemporary art market repertoire. This originally independent art currently found in the private sector is what is known internationally as "Urban Art".

Historically, the birth, evolution and crossing of borders of practices from public spaces to their inclusion in galleries are relatively recent events. However, much has occurred from the first exhibitions of the 1970s to the present day. This article will look at everything that has happened since then, showing how the inclusion of works by graffiti writers and street artists in the commercial art market has influenced the art and its conservation.

Key words: auction, street art, graffiti, urban art, galleries, independent public art, art-market

\section{Introducción}

Desde las primeras muestras de grafiti en el metro de Nueva York a finales de los 60 y principios de los 70 del siglo XX (Castleman, 1982: X), seguida de su rápida expansión en otros soportes de la ciudad y la ampliación de fronteras en la exposición y accesibilidad de éste junto al arte urbano en los 80 (Schacter, 2013: 16), los movimientos de arte independiente han tomado posesión del espacio público como el lugar y soporte donde estas formas de arte se crean, desarrollan, exponen y conviven.
El arte localizado en estos entornos se denomina arte público independiente (Abarca Sanchis, 2010: 39-44) y está liderado por los movimientos del grafiti y el street art o arte urbano [1]. Ambas formas con estilos paralelos el uno del otro, han empleado el espacio público como lienzo en blanco, un perfecto soporte para exponer gratuitamente conceptos y formas a los diferentes públicos. En cualquier caso, la relación de sus trabajos con el público puede variar dependiendo de la accesibilidad a la obra, la intencionalidad del artista, el interés del público o simplemente, el mensaje expuesto y su vinculación al entorno. 
El empleo de la calle de forma libre por los artistas no supone la limitación de su práctica artística en un único entorno. Al igual que los artistas emplean cualquier soporte o parte del mobiliario urbano en el espacio público, participan en eventos organizados vinculados al grafiti como Meeting of Styles (festival celebrado internacionalmente y centrado en el grafiti mural desde 1997) (Gerullis, 2013: 5-7), u otros eventos con escritores de grafiti y artistas urbanos como The Wynwood Walls en Miami (Wynwood Walls, 2019), Upfest en Bristol (Upfest, 2019) o Nuart en Stavanger y Aberdeen (Nuart, 2019); sus fronteras también se expanden abarcando espacios privados. Es de este modo como artistas vinculados al grafiti y el arte urbano hacen uso, al igual que otros artistas contemporáneos, de espacios más tradicionales o institucionalizados, y sectores comerciales, para exponer su obra. [figuras 1y 2]

\section{Primeras exposiciones en galerías}

Entre los años 70 y 80 del siglo XX, y al mismo tiempo que el grafiti se desarrollaba en Nueva York y se expandía por EEUU y Europa, se celebraron las primeras exposiciones vinculadas con el movimiento y sus practicantes. En algunos casos promovidas por asociaciones relacionadas

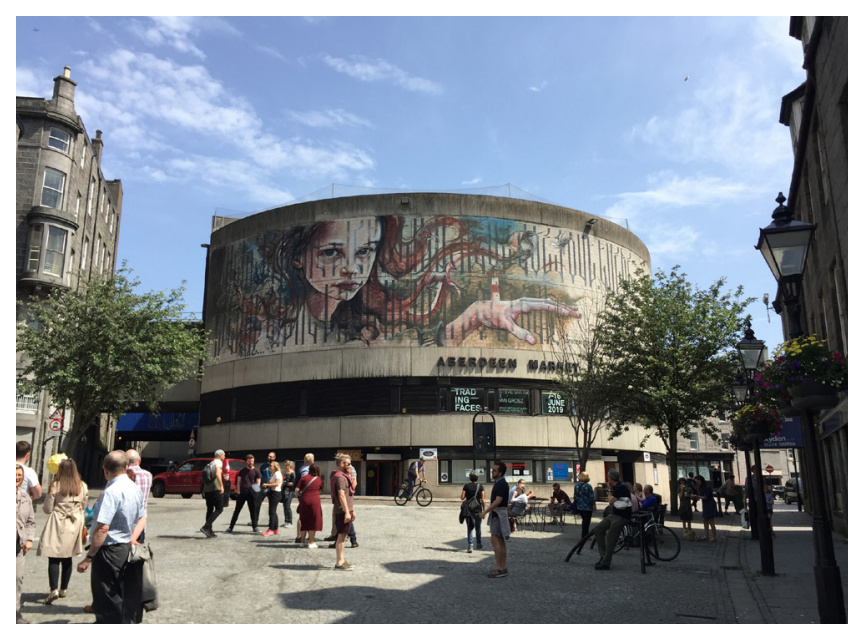

Figura 1. Intervención de Herakut en Nuart, Aberdeen, edición 2017.

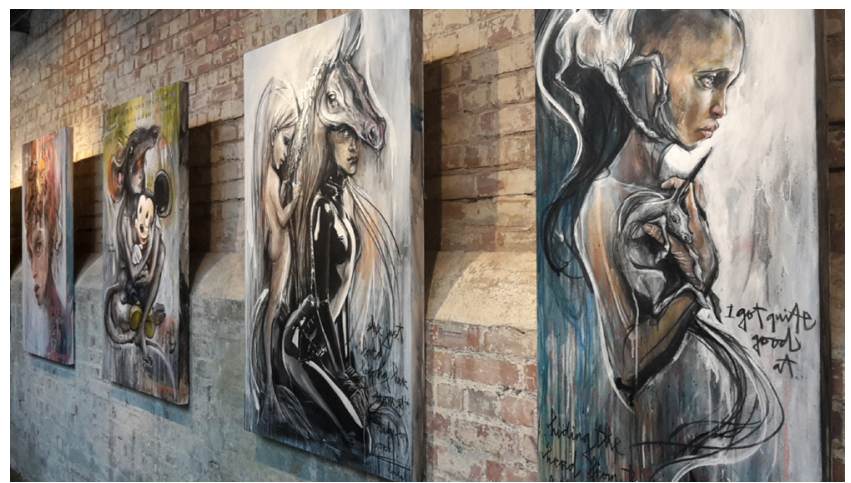

Figura 2. Exposición de Herakut en StolenSpace Gallery, Londres, 2017. con los escritores de grafiti y en otros casos, por galeristas externos que apreciaban lo que estaba sucediendo en las calles de Nueva York.

Por un lado, la organización de asociaciones de escritores de grafiti como United Graffiti Artists (UGA) y Nation of Graffiti Artists (NOGA), surgidas en 1972 y 1974 respectivamente, ayudó en la entrada a escritores de grafiti en círculos artísticos convencionales. Los objetivos de estas asociaciones eran: descriminalizar la práctica, ayudar a aquellos practicantes en entornos de exclusión social, fomentar la práctica artística entre los mismos y proveer un medio en el que dar a conocer sus obras al público. Todo ello mediante la organización de grupos, eventos y medios de difusión. En general, agrupaban a escritores que habían mostrado su potencial artístico en las calles, al mismo tiempo que los ayudaban en su producción en otros entornos, como los estudios de artista.

Por otro lado, fuera de esas asociaciones con fines creativos y de difusión, también se organizaron exposiciones que contaban con escritores de grafiti y otros artistas independientes que no trabajaban directamente con asociaciones. La primera exposición que reunía escritores de grafiti es la Razor Gallery del SoHo en septiembre de 1973, seguida de la Detective Show en 1978, una exposición al exterior de artistas procedentes de prácticas callejeras (Lewisohn, 2009: 15). Pero no fue hasta 1981 cuando se realizó otra exposición con el título Street Art, organizada por el Washington Project of the Arts, como otro intento de reunir "artistas trabajando puramente en el entorno urbano e ignorados por la institución del arte" (Lewisohn, 2009: 93). Y, la exposición, Post-graffiti en 1983, que presentaba a artistas trabajando "más allá del grafiti" (Janis y Neumann, 1983) incluyendo a Keith Haring que, aunque externo al grafiti (Gablik, 1982), sus intervenciones en el espacio público y el metro de Nueva York se consideran precursoras del arte urbano que conocemos hoy en día (Lewisohn, 2019: 94-96) [figura 3].

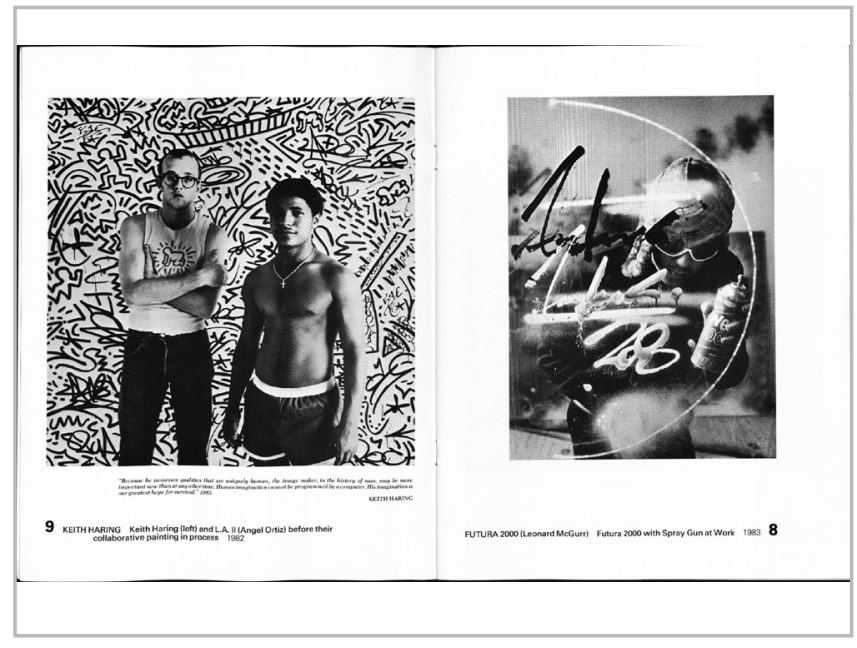

Figura 3. Catálogo de exposición Post-graffiti. 
De este modo fue como el mundo del arte abrió camino a algunos artistas que, con una corta carrera a nivel temporal -pero completa a nivel práctico en el grafiti generalmente-, buscaban nuevas maneras en la difusión de su arte. Ellos encontraron su forma de expresión plástica y utilizaron lo aprendido en las calles, y lo que consideraban su marca (su estilo), para forjarse una profesión como artistas. Al mismo tiempo, estos eventos aportaban a los artistas cierto reconocimiento y seguridad en su trabajo, lo cual no ocurría siempre al intervenir en la calle. Al mismo tiempo, la participación en exposiciones, grupos de trabajo, así como encargos decorativos, resultaba un buen medio para sufragar y continuar su práctica artística, lo cual sigue vigente hoy en día.

Respecto al público, el interés, en un principio minoritario, fue expandiéndose poco a poco, desde un entorno cercano a sus practicantes, hacia un público más general. Y lo mismo para los galeristas y coleccionistas que veían el potencial de los escritores de grafiti y artistas. Un claro ejemplo de ésto fue Martin Wong, apasionado de esas formas de arte independiente surgidas en Nueva York, el cual recogió material sobre grafiti durante toda su vida y donó a los fondos del Museum of the City of New York en 1994 (McCormick y Corcoran, 2003: 7). Esta donación de fondos se considera la inclusión más importante de grafiti en un museo hasta la fecha. La colección fue expuesta en 2014 en el mismo museo en la exposición City as Canvas (Museum of the City of New York, 2014).

De este modo, el interés del público y el apoyo recibido por algunos artistas, fue notablemente importante en la futura consideración de sus obras, y con ello, la expansión en otros sectores expositivos.

\section{Expansión del grafiti y el arte urbano, y el surgimiento del Urban Art}

A pesar de mantenerse en el radar de ciertos especialistas, no fue hasta los años 90 cuando el arte público independiente realmente despertó interés en círculos más generales. Fue cuando las formas de arte público que recordaban al grafiti pero que mostraban diferencias en estilo y una diversidad notable en los mensajes se expandió internacionalmente. Después de muchos intentos en el uso de terminología para identificar estas formas, como post-graffiti (Abarca Sanchis, 2010: 54-56; Waclawek, 2011: 7,12,29; García Pardo, 2015: 52-54), el término street art a nivel internacional y arte urbano en España, se empleó para designar esas muestras de arte alternativo en el entorno público (Schacter, 2013: 16) que no fueran grafiti.

Entre el arte urbano y el grafiti siempre ha existido cierta vinculación, y aun hoy en día hay confusión a la hora de diferenciar ambos movimientos. Esto es generalmente debido a que muchos de los artistas urbanos comenzaron inicialmente a explorar el entorno público como escritores de grafiti (Waclawek, 2011:7), pero expandieron sus fronteras más allá de la práctica posteriormente. No obstante, hay que entender que la terminología puede variar debido a la cercanía en el tiempo, pero en parámetros generales ayuda a la diferenciación de ambos movimientos, mostrando adecuación y respeto a los artistas acorde a los principios establecidos en cada manifestación, y con ello, también con los términos o vinculaciones que cada artista decide o asume para exponer su arte en el espacio público.

El interés generado por el público está, en gran medida, provocado por especialistas y amantes de estas formas de arte alternativo y generalmente subversivo, lo cual ayudó directamente a la inclusión del grafiti y el arte urbano en el museo. Las primeras exposiciones de carácter temporal, intentaron explicar lo que estaba ocurriendo en la calle, resaltando los mensajes y la nueva dinámica de conceptos que se exponía al recuperar el espacio urbano como lugar para la muestra de arte puramente público, y donde cualquier persona podía ser partícipe del arte que se encontraba. Pero, al mismo tiempo, mientras fuentes documentales como el vídeo y la fotografía resultaban muy convenientes para exponer tales realidades en el entorno del museo, las obras expuestas dentro de éste combinaban el uso de la práctica mural con obras en caballete, láminas y esculturas, entre otras.

Uno de los eventos más importantes relacionados con este hecho fue la exposición Street Art en el museo Tate Modern de Londres en 2008. Street Art fue la primera exposición temporal de esta índole realizada en un museo público. Comisionada por el especialista en grafiti y arte urbano, Cedar Lewisohn, la exposición hacía una retrospectiva de las prácticas independientes a nivel histórico y formal, mostrando obras de artistas ya reconocidos en los movimientos, junto a obras de gran influencia para escritores de grafiti y artistas urbanos. Al mismo tiempo que en el interior del museo se exhibían obras en soporte móvil, la fachada principal del edificio mostraba las obras murales de cinco artistas internacionales: Blu, Faile, JR, Nunca, Os Gemeos y Sixeart (Tate Modern, 2008ab). Además, como guiño a la importancia de la exposición en el panorama artístico del momento, entre 2008 y 2009 , el grafiti como movimiento y el nombre de escritores originarios de éste, como Taki 183, Blade, Seen y Lee Quiñones, fueron introducidos dentro de la línea temporal de movimientos del arte moderno y contemporáneo diseñada por Sara Fanelli. [figuras 4 y 5]

El siguiente evento, mayor en obra y en artistas participantes, fue la exposición Art In The Streets en el Museum of Contemporary Art de Los Angeles (MoCA) en 2011. Esta exposición fue comisionada por el director del museo Jeffrey Deitch junto a los especialistas Roger Gastman y Aaron Rosepor. En ésta, el recinto de exposiciones The Geffen Contemporary at MoCA en 


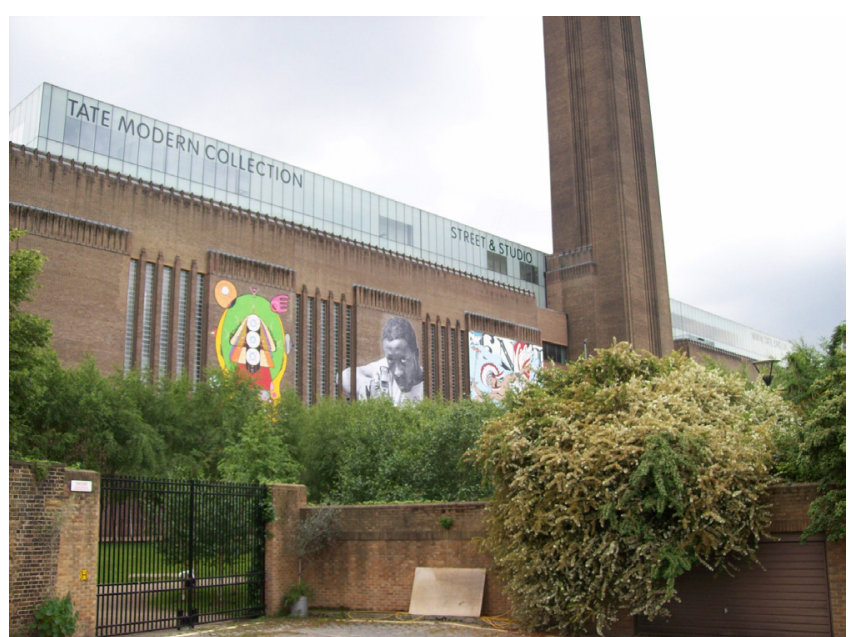

Figura 4. Fotografía de las intervenciones en la fachada de la Tate Modern, Mayo 2008.

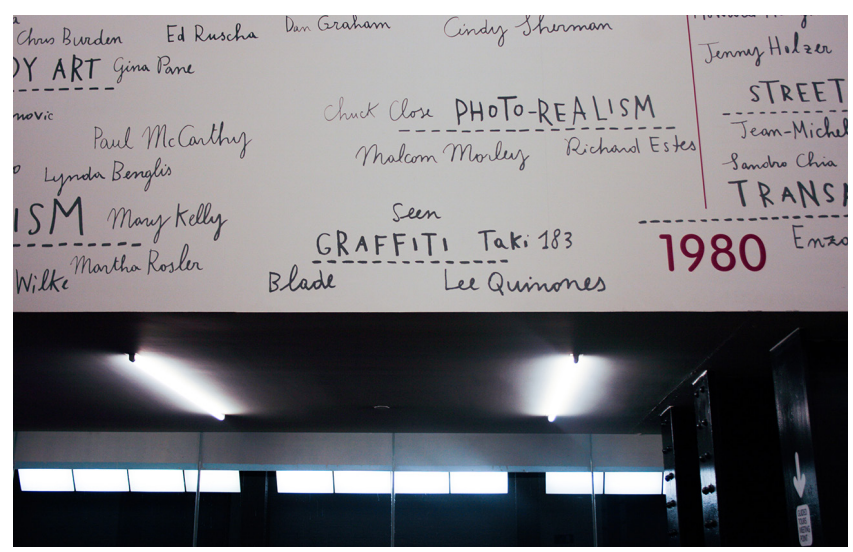

Figura 5. Fotografía de la línea temporal de Sara Fanelli, detalle grafiti.

Downtown Los Angeles reunió a un total de cincuenta escritores de grafiti, artistas urbanos y fotógrafos (MoCA, 2011).

Otras exposiciones han seguido muy de cerca estos eventos en otros entornos, con gran magnitud y público interesado en los mismos, más allá de los museos públicos. En la actualidad son muchas las galerías especializadas, casas de subasta, ferias de arte y festivales, así como museos, los que ofrecen en su catálogo obras que se vinculan o recuerdan al grafiti y el arte urbano, pero ¿es correcto usar estos términos para obras realizadas específicamente para el sector privado? En parámetros generales, la diversidad de las obras con otros estilos artísticos, y específicamente, la necesidad de separar la terminología de las obras de arte público independiente de aquellas obras realizadas para sectores más comerciales, han producido el uso de términos diferenciados en cada caso. Del mismo modo que existe una diferenciación entre el grafiti y arte urbano como movimientos independientes uno del otro, también ha surgido una diferenciación terminológica en aquellas obras que aunque vinculadas a estos movimientos, se presentan fuera del espacio público. Es de este modo como el relativamente reciente término Urban art ha entrado dentro de la dinámica. Este término se emplea principalmente para identificar obras procedentes o que recuerdan al grafiti y arte urbano, o que generalmente, aunque no siempre, son realizadas por escritores de grafiti y artistas urbanos para la galería, y que en cualquier caso son accesibles exclusivamente en sectores privados (Lewisohn, 2011: 13; Young, 2014: 9).

Como consecuencia a la inclusión y expansión de obras vinculadas a prácticas de arte independiente dentro del espacio museístico, otros eventos similares han ocurrido en los últimos años. Uno de los eventos expositivos con mayor magnitud en la organización y exposición de obras del Urban art ha sido Beyond the Streets. La primera exposición vinculada a este título fue celebrada en Los Angeles en 2018, seguida por otra en Nueva York en 2019. Ambas comisionadas por Roger Gastman, esta tipología de evento reúne obras y artistas emblemáticas del grafiti, arte urbano y más allá, en casi 9300 metros cuadrados de suelo expositivos con más de ciento cincuenta artistas participantes en su segunda edición (Beyond the Streets, 2019) [figura 6].

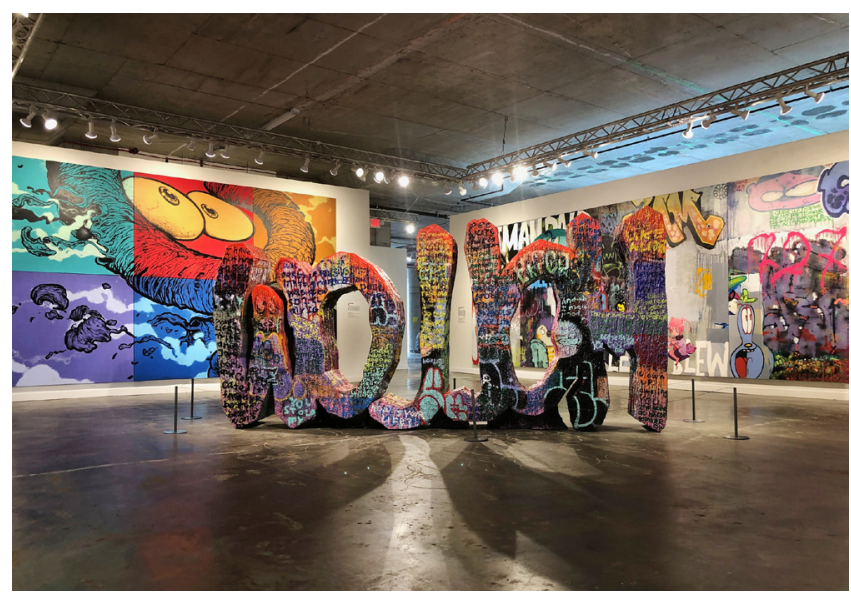

Figura 6. Exposición Beyond the Streets, en Nueva York, 2019.

\section{El artista independiente en el sector privado}

La introducción de estas formas de arte, o de los propios escritores de grafiti y artistas urbanos en tales espacios, no significa la pérdida de identidad de sus obras "libres" en entornos "normalizados" o "pseudo-institucionalizados", sino que se trata de otras obras estéticamente muy similares, pero que conceptualmente suelen mostrar diferencias. A pesar de que muchos galeristas especializados en el Urban Art animan a los artistas independientes a trasladar su arte público al sector galerístico sin limitaciones, el mercado del arte o simplemente el entorno privado puede cambiar singularmente el concepto inicial de la obra.

En muchos casos, artistas que trabajan en el espacio público libremente y en la galería, hacen distinción de su obra en cada entorno empleando diferente terminología como Wow123 - Graffiti writer/Versatil Artist - (Genesius, 2017), o Conor Harrington - Inside/Outside - (Harrington, 
2019) entre muchos otros. El entorno de exposición supone un condicionante importante para aquellos artistas que trabajan el concepto de su obra y defienden la libertad de expresión en el espacio público, y las formas originales del grafiti y el arte urbano. En general, muchos de estos artistas hacen cambios no solo en las imágenes empleadas, sino también distinción en los mensajes expuestos o simplemente empleando una intencionalidad diferente, ya que al mismo tiempo que, en el espacio público, el espectador encuentra la obra sin una intención directa de encontrarla; en la galería, el espectador tiene una intención directa de encontrar la obra de un cierto artista. Por todo ello, la relación entre la obra y el público cambia en el momento que el entorno es diferente, y con ello el artista adapta estos parámetros en cada caso. [figura 7].

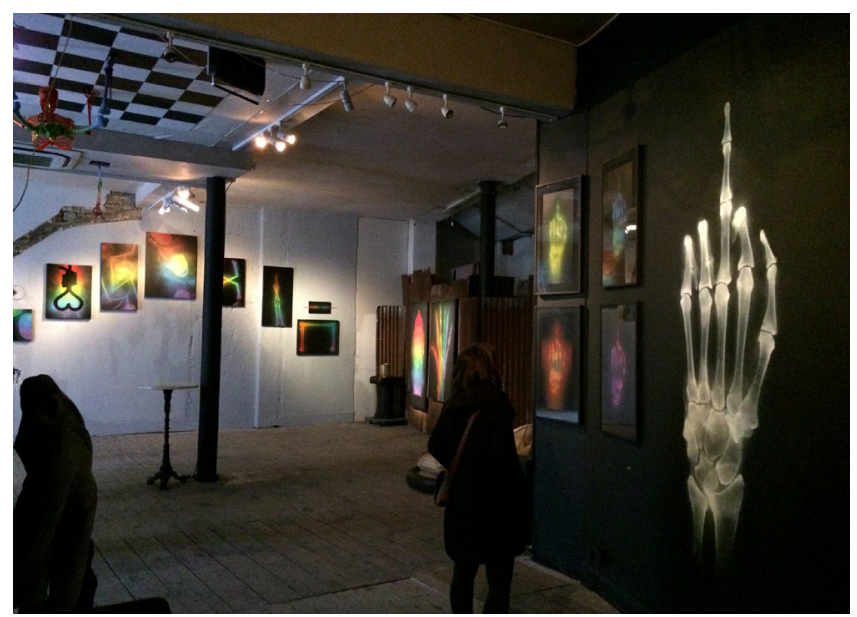

Figura 7. Exposición de Shok1 en Londres, 2013.

Respecto a la opinión de los artistas sobre su participación en exposiciones y la venta de sus obras, existen muchas diferencias y posturas. Por un lado, aquellos que focalizan su carrera en la producción artística, se presentan más abiertos a la introducción de sus obras en el mercado del arte, aceptando y defendiendo la necesidad de emplear la galería y la calle como dos espacios diferenciados. Así pues, artistas como JonOne han subrayado la importancia de tener una galería que los represente o muestre interés por su obra y señala que las galerías "intentan maximizar el potencial" de los artistas (Boujnah y Gabaret, 2015a). No obstante, y por otro lado, otros artistas como C215, reniegan parcialmente de esa necesidad, ofreciendo sus trabajos a la venta en sus propias páginas web, mostrando galerías actualizadas y abiertas a todos (Boujnah y Gabaret, 2015b).

\section{Las casas de subasta y los artistas}

Con la aceptación de obras alternativas dentro del entorno comercial, y el incremento tanto entre seguidores como en la demanda por poseer obras de ciertos artistas, las casas de subasta se introdujeron como partícipes en este intercambio. A pesar de que no hay un evento particular donde se pueda señalar el inicio de las casas de subasta tratando obras relativas al Urban art, algunas de las primeras subastas públicas con obras relacionadas datan de 2005 (Sotheby's, 2019a).

Así, la introducción del Urban Art en casas de subastas fue paulatina, con la presencia de obras puntuales en ciertas subastas mezcladas con obras de arte contemporáneo, o subastas de colecciones de arte de coleccionistas específicos. Gracias al notable interés revelado en la última década, la venta de este tipo de obras en casas de subasta ha creado mayor atención y crecimiento, no solo incluyendo obras del Urban Art dentro de este sector, sino también creando eventos específicos para la venta de las mismas. Como ocurrió con Against the Wall, dentro de la subasta Contemporary Curated celebrada en noviembre 2018, que contó con nueve trabajos donados por artistas del Urban Art, y cuyos beneficios fueron a la organización sin ánimo de lucro Movember (Sotheby's, 2018); o también, y más reciente, Banksy / Online, celebrada en septiembre de 2019, la primera venta online de impresiones exclusivamente del artista (Sotheby's, 2019b).

Igualmente y en consecuencia al incremento de interés, en la actualidad, casas de subasta como Christie's, Phillips y Sotheby's ya incluyen las obras de cierta manera vinculadas a artistas alternativos o que recuerdan al grafiti y arte urbano dentro de sus departamentos de arte de posguerra, arte del siglo XX y arte contemporáneo (Christie's, 2019; Phillips, 2019a; Sotheby's, 2019c) mientras que otras las presentan independientemente con el término urban art (Chiswick Auctions, 2019; Art Curial, 2019).

Respecto a la participación de los artistas en estos eventos, su presencia es en general anecdótica y son muchos los que tras producir de forma libre en el espacio público ven la compra-venta de obras en entornos extremadamente privatizados como algo contraproducente o simplemente basado en un círculo ficticio externo a la idea romántica del arte para el deleite. Un ejemplo es Blek Le Rat, quien menciona en una entrevista para Street Art À la Française, que el incremento de los precios en las subastas es algo ficticio y en muchas ocasiones realizado de forma fortuita y algo picaresca (Boujnah y Gabaret, 2015a).

Paralelamente a las subastas de obras en papel, lienzo o esculturas y directamente realizadas bajos las expectativas del Urban Art, también existen casos de subastas de obras del grafiti y arte urbano, originalmente realizadas en el entorno público. Esto, en algunos casos, ha enfrentado notablemente el uso y la explotación de las mismas con la intención primera de las obras, la exposición y acceso libre.

El artista que ha despertado mayor revuelo en estas subastas ha sido Banksy, aunque no de forma directa. En su caso, la fama que existe detrás de su anonimato y el carácter de sus obras ha influenciado notablemente también en la venta de las intervenciones que realiza en el espacio abierto. En Reino Unido, donde ha ocurrido la mayoría de estas situaciones, se establece como lícito la compra-venta de 
obras localizadas en el espacio urbano, sin necesidad de un permiso del artista. Específicamente, la legislación señala que el dueño del edificio donde se encuentra una obra realizada sin contrato o comisión, posee los derechos de compraventa de la misma (Laker Legal, 2013). Por lo que, aunque la propiedad intelectual la posea el artista (Reino Unido, 2017), este siempre quedará en segundo lugar en esos casos, y todas aquellas obras realizadas con o sin permiso explícito sobre tales espacios, quedan al amparo del propietario.

Esto ha favorecido la venta de obras en soporte mural o esculturas, no solo por casas de subasta sino también por empresas privadas que han superpuesto el valor económico de las obras sobre cualquiera de los otros valores (artístico, histórico, social, antropológico) que las mismas pudieran tener en el espacio para las que fueron creadas. El estamento de empresas que fomentan este tipo de acciones se basa en salvar, restaurar y vender las obras para evitar el vandalismo y multas, como apunta The Sincura Group (Baxter, 2019). En general, Banksy no ha mostrado un posicionamiento reaccionario directo respecto a estas situaciones [2], pero sí que ha apoyado casos muy puntuales donde la compraventa de sus obras en el espacio público podían suponer un beneficio para la comunidad o propietarios en situaciones delicadas, como la venta de Mobile Lovers para salvar un club juvenil en Bristol (The Telegraph, 2014).

Otro caso a destacar sobre la relación de artistas independientes y casas de subasta es Stik. Este artista británico ha mostrado un gran interés y apoyo en la venta de sus obras en estos entornos, sean realizadas para su venta en sectores privados o en casos donde la obra, originalmente realizada en el espacio público de forma libre, termina en la casa de subasta y posteriormente en sectores privados, siempre y cuando los beneficios se destinen a causas sociales. Las subastas de las obras del segundo caso suelen estar apoyadas por el artista y por asociaciones con fines benéficos, como es el ejemplo de las subastas organizadas en diciembre 2016 y diciembre 2018 por Phillips Auctions, donde las obras fueron autentificadas por el artista y sus ventas aprobadas por él mismo. Los beneficios obtenidos fueron directamente a las asociaciones locales, como la subasta de la obra Magpie cuyos beneficios fueron a parar a Magpie Social Centre en Bristol (Phillips, 2019b); o la subasta de un fragmento de la obra Big Mother, cuyos beneficios fueron destinados a Artification Charity, un programa gratuito para la promoción del arte entre los residentes de Charles Hocking House en el barrio de Acton en Londres (Phillips, 2019c) [figuras 8 y 9].

No obstante, Stik ha sufrido también la apropiación de algunas de sus obras en el espacio urbano, como un caso en Hackney, Londres (Stik, 2019a), así como la aparición de dichas obras en casas de subastas, sin su aprobación (Hackney Citizen, 2019). En cualquier caso, el artista ha promovido la recuperación de tales obras, y en otros casos ha establecido acciones extremas para evitar la desaparición y venta no autorizada de las mismas, como en el caso de Gdansk, Polonia (Stik, 2019b).

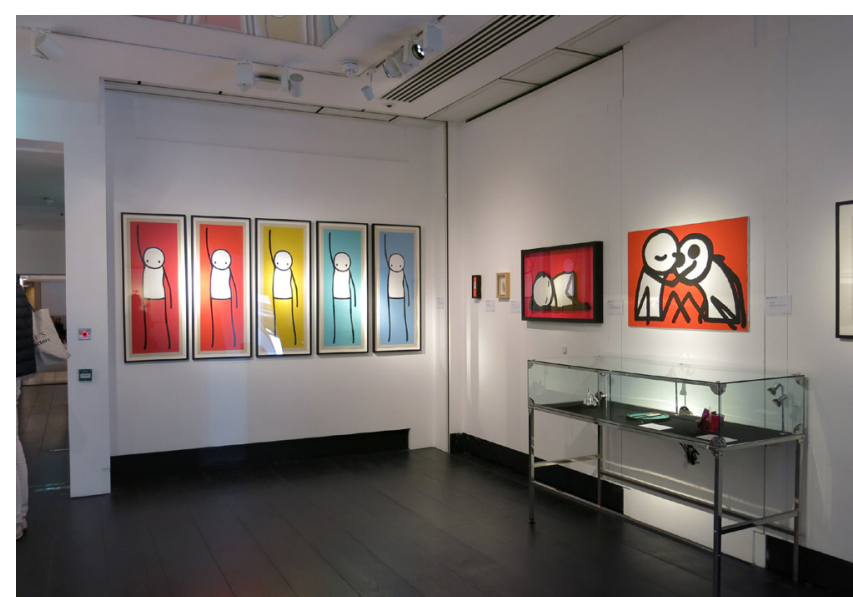

Figura 8. Obras de Stik en la subasta Prints and Multiples en Christie's, Londres, 2019.

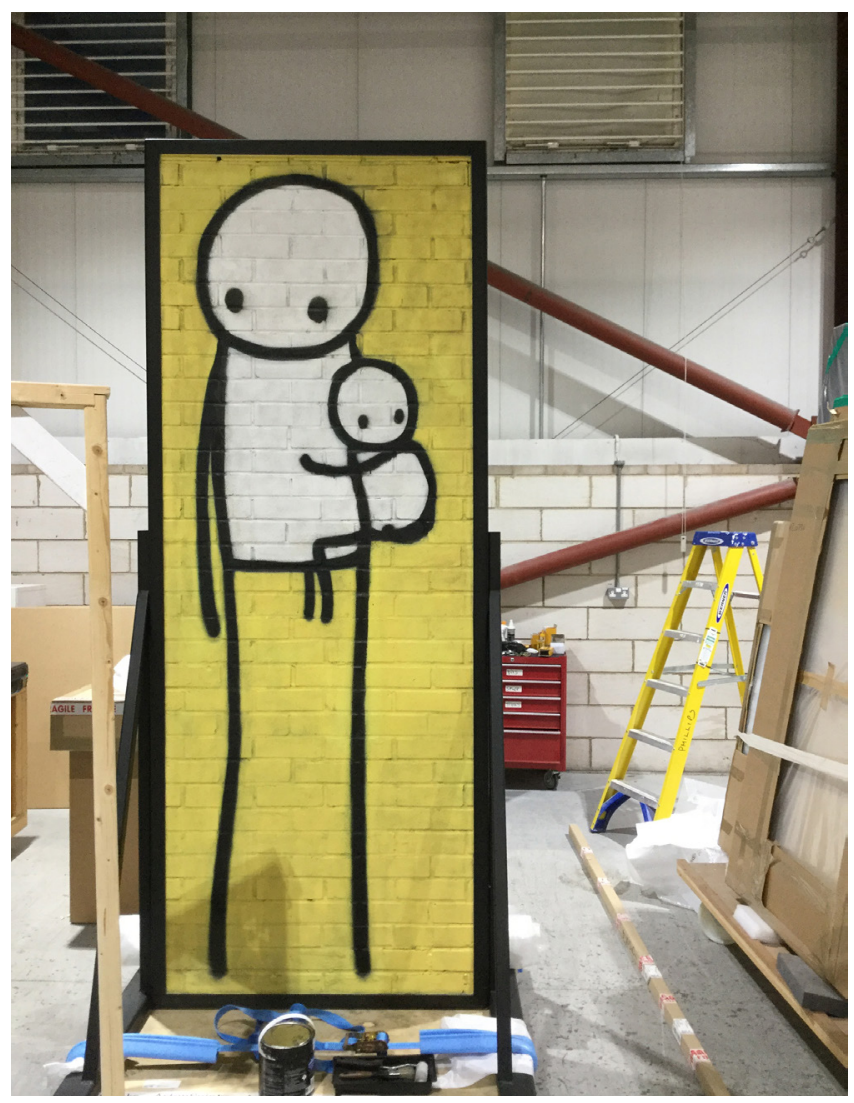

Figura 9. Big Mother de Stik, previamente a la subasta en Phillips, 2018.

\section{Conclusiones}

La inclusión de manifestaciones de arte público independiente dentro de entornos institucionalizados o incluso dentro del mercado del arte, resulta positivo para los artistas y para el público. Esto ha generado un mayor interés y seguimiento en artistas procedentes de sectores menos reconocidos y que han trabajado para la recuperación del entorno urbano como lugar de trabajo, exposición y para el arte (realmente) público; y el empleo de la crítica desde una perspectiva más alternativa y menos esperada. 
No obstante, las diferencias conceptuales y creativas de los artistas vinculados a movimientos independientes y sus obras, al ser introducidos en el sector comercial, varían notablemente. Por ello, es importante establecer una diferenciación terminológica entre las obras realizadas para fines comerciales y las relacionadas por movimientos independientes o por artistas. El empleo del término Urban Art mantiene una separación entre lo que está hecho para la venta y lo que simplemente está hecho para el disfrute sin comisiones o influencias externas (arte público independiente). Al mismo tiempo, la introducción de artistas originalmente vinculados al grafiti y arte urbano dentro de los sectores comerciales supone un punto positivo para los artistas y sus obras, ya que favorece la continuidad de la producción dentro y fuera del sector galerístico. Asimismo permite, en parámetros generales, establecer diferencias entre las obras y ampliar conceptos y posibilidades; y también, refuerza la identidad libre de las obras realizadas en el espacio urbano, ampliando las fronteras de cierto público focalizado anteriormente solo en sectores comerciales o institucionalizados.

Con la expansión de obras de artistas vinculados al grafiti y arte urbano dentro del mercado del arte, se han dado ciertas situaciones que confunden el arte para uso comercial y el simplemente hecho para el disfrute en el entorno público. Esto ocurre promovido por un interés puramente especulativo, donde los límites que se establecen gracias a la identificación terminológica y a las diferencias entre los espacios de trabajo se sobrepasan sin seguir una ética correcta, obviando los valores, conceptos e identidades originalmente mostradas por los artistas. Por ello, aquellas obras que no fueron realizadas para un sector privatizado, no deberían terminar en tales espacios sin el consentimiento del artista y de la comunidad, ya que el valor económico nunca debe anteponerse al establecimiento de un procedimiento ético o moral consensuado por todas las partes interesadas o vinculadas a la obra.

Por otro lado, y para concluir, es importante subrayar que el incremento en la producción en sectores más comerciales implica la necesidad de establecer patrones de conservación que en cualquier caso pueden ser aplicados en entornos privados pero también en entornos públicos. Pese a que los factores de degradación en las obras pueden variar dependiendo si la obra se encuentra en espacio abierto o en entorno pseudo controlado, la producción y materiales empleados por los artistas en ambos entornos se asemeja y, en muchos casos, incluso, puede ser idéntica. Esto es inherentemente positivo para los especialistas en conservación que han trabajado e investigado en el establecimiento de mecanismos de conservación sobre técnicas empleadas en el grafiti y arte urbano. Desde una postura positiva, se valora como favorable la demanda comercial de obras del Urban Art ya que implica un mayor interés e inversión por desarrollar tales mecanismos en cualquier campo. Con ello, se espera que las propuestas de investigación y las posibilidades de conservación, actualmente escasas en producción, aumenten notablemente en los próximos años.

\section{Notas}

[1] Street Art es la acepción inglesa original para expresar formas de arte independiente en el espacio público que van más allá del empleo del nombre y de los soportes de la ciudad como generalmente hace el grafiti. El término en español para esta forma de expresión es arte urbano, término que no debe confundirse con Urban art (vinculado al sector comercial).

[2] La plataforma Pest Control (Pest Control, 2019) de Banksy, ofrece un servicio de autentificación de obras realizadas para el disfrute privado, como impresiones en papel y pinturas sobre lienzo. El artista ofrece este servicio como medio para evitar fraudes, pero éste no se ofrece para casos de obras extraídas del entorno urbano, las cuales no son comúnmente autentificadas.

\section{Bibliografía}

ABARCA SANCHIS, F.J. (2010). El postgraffiti, su escenario y sus raíces: graffiti, punk, skate y contrapublicidad. Tesis doctoral. Madrid: Universidad Complutense de Madrid. http://eprints.ucm. es/11419/ [Consulta: 29 de agosto de 2019]

BAXTER, A (2019). The controversial Banksy Book. http:// banksybook.com/ [Consulta: 26 de agosto de 2019]

BOUJNAH, S; GABARET, J. (2015a) Street Art À la Française, Episodio 4 "Wall Street Art".

BOUJNAH, S.; GABARET, J. (2015b) Street Art À la Française, Episodio 5 "The Art of Marketing".

CASTLEMAN, C.(1982). Getting Up. Subway Graffiti in New York. Massachusetts: The MIT Press.

GABLIK, S.(1982). "Report From New York: The Graffiti Question". En Art in America Magazine, archives. https://www. artinamericamagazine.com/news-features/magazines/from-thearchives-report-from-new-york-the-graffiti-question/ [Consulta: 29 de Agosto de 2019]

GARCIAPARDO, B. (2015).Grafitiy Postgrafitien laciudaddeValencia: una perspectiva crítica. Tesis doctoral. Valencia: Universidad de Valencia. http://roderic.uv.es/handle/10550/45854 [Consulta: 26 de agosto de 2019]

GENESIUS, M. (2017). Markus Genesius, Wow 123. http://markusgenesius.com/ [Consulta: 13 de marzo de 2017]

GERULLIS, M.(2013). Meeting of Styles, Volume 1. Berlin: From Here to Fame.

HACKNEY CITIZEN (2019). "Stik's community mural 'finally' back in Poland after long tug-of-war with London gallery". En 
Hackney Citizen, June 21, 2019. https://www.hackneycitizen. co.uk/2019/06/21/stik-community-mural-back-poland/ [Consulta: 26 de agosto de 2019]

HARRINGTON, C.(2019). Conor Harrington - Works. http://www. conorharrington.com/[Consulta: 29 de agosto de 2019]

JANIS, S. y NEUMANN, D. (1983). Post-graffiti: exhibition by postgraffiti artists, opening Thursday December 1-31st, at Sidney Janis Gallery, New York, 1983. Nueva York: The Gallery.

LAKER LEGAL (2013). "Banksy graffiti, Intellectual Property \& who owns what?". En Laker Legal Solicitors Blog. http://www. lakerlegal.co.uk/banksy-graffiti-intellectual-property-who-ownswhat/ [Consulta: 26 de agosto de 2019]

LEWISOHN, C. (2009). Street Art. The Graffiti Revolution. Londres: Tate Publishing.

LEWISOHN, C. (2011). Abstract graffiti. Londres: Merrell.

McCORMICK, C. y CORCORAN, S. (2013). City as Canvas: New York City Graffiti from the Martin Wong Collection: New York City Graffiti and the Martin Wong Collection. Nueva York: Skira Rizzoli.

MOCA (2016). "Art in the Streets". En MoCA website. https://www. moca.org/exhibition/art-in-the-streets [Consulta: 26 de agosto de 2019]

MUSEUM OF THE CITY OF NEW YORK (2014). "City as Canvas. February 4 - September 21, 2014". En Museum of the City of New York, Exhibitions. http://www.mcny.org/exhibition/city-canvas [Consulta: 26 de agosto de 2019]

NUART (2019). Nuart Festival. http://www.nuartfestival.no/home [Consulta: 29 de agosto de 2019]

PEST CONTROL (2019). Pest Control Handling Services http:// pestcontroloffice.com/ [Consulta: 26 de agosto de 2019]

PHILLIPS (2019a). "20th Century and Contemporary Art", En Phillips Auctions, Departments. https://www.phillips.com/ departments/department?DepartmentName=Contemporary [Consulta: 26 de agosto de 2019]

PHILLIPS (2019b). “Stik, Magpie, 2009. Lot 90" En Phillips - London Auction 8 December 2016. https://www.phillips.com/detail/stik/ UK010816/90 [Consulta: 26 de agosto de 2019]

PHILLIPS (2019c). "Stik, Big Mother, 2014. Lot 14" En Phillips London Auction 5 December 2018. https://www.phillips.com/ detail/stik/UK010818/14 [Consulta: 26 de agosto de 2019]

Reino Unido (2017). "Copyright, Designs and Patents Act 1988". En Acts of Parliment, 15 de noviembre de 1998 [versión actualizada 10/7/2017], chapter 48, article 11.

SCHACTER, R. (2013). The World Atlas of Street Art and Graffiti. Londres: Aurum Press.
SOTHEBYS (2018). "Contemporary Curated". En Sotheby's auctions, results. http://www.sothebys.com/en/auctions/2018/ contemporary-curated-I18027.html?locale=en [Consulta: 26 de agosto de 2019]

SOTHEBYS (2019a). "Banksy, Untitled (Mother and Child), Lot 408". En Sothebys Auctions, Contemporary Art, E-catalogue 2005. https://www.sothebys.com/en/auctions/ecatalogue/2005/ contemporary-art-w05709/lot.408.html [Consulta: 26 de agosto de 2019]

SOTHEBYS (2019b) "Banksy | Online". En Sothebys Auctions, Upcoming. $<$ https://www.sothebys.com/en/auctions/2019/ banksy-online-sale-I19357.html?locale=en [Consulta: 29 de agosto de 2019]

SOTHEBYS (2019c). "Contemporary Art". En Sotheby's auctions, Departments. https://www.sothebys.com/en/departments/ contemporary-art?locale=en [Consulta: 26 de agosto de 2019]

STIK (2019a). "Stolen $£ 30,000$ Stik Sculptures Returned to Community". En Stik website. http://stik.org/news [Consulta: 26 de agosto de 2019]

STIK (2019b). "STIK Deletes Remains of Missing Artwork". En Youtube, Stik Stik channel. https://www.youtube.com/ watch?v=G0oRvx-n1n0 [Consulta: 26 de agosto de 2019]

TATE MODERN (2008a). "Street Art Exhibition". En Tate website. https://www.tate.org.uk/whats-on/tate-modern/exhibition/ street-art [Consulta: 29 de agosto de 2019]

TATE MODERN (2008b). "Street Art Exhibition, Press Releases". En Tate website. https://www.tate.org.uk/press/press-releases/ street-art-tate-modern [Consulta: 29 de agosto de 2019]

THE TELEGRAPH (2014). "Banksy artwork saves youth club as it sells for $£ 400 k "$. En The Telegraph, Culture, Art - 24 August 2014. https://www.telegraph.co.uk/culture/art/11059481/Banksyartwork-saves-youth-club-as-it-sells-for-400k.html [Consulta: 29 de agosto de 2019]

UPFEST (2019). Upfest 2016. Europe's largest Street Art and Graffiti Festival. http://www.upfest.co.uk/[Consulta: 29 de agosto de 2019]

WACLAWEK, Anna. (2011). Graffiti and Street Art. Londres: Thames and Hudson.

WYNWOOD WALLS (2019). The Wynwood Walls' website. http:// www.thewynwoodwalls.com/ [Consulta: 29 de agosto de 2019]

YOUNG, A. (2014). Street Art, Public City. Law, Crime and the Urban Imagination. Oxon: Routledge. 


\section{Autor/es}

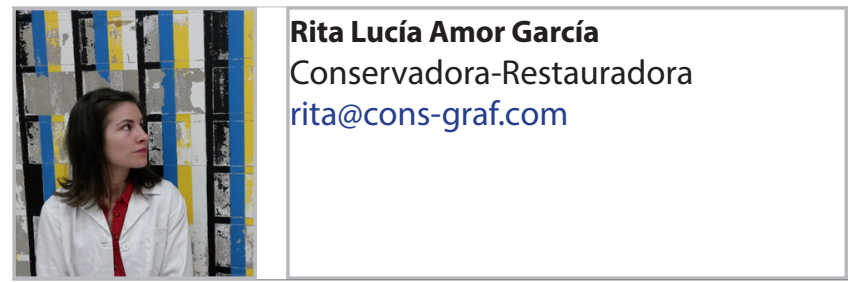

Es Doctora en Ciencia y Restauración del Patrimonio Histórico-Artístico. Trabaja en Londres como conservadora especialista en arte contemporáneo y urban art, y como técnica de exposiciones. Es también licenciada en Bellas Artes y posgraduada en Conservación y Restauración de Bienes Culturales, ambos por la Universitat Politècnica de València (UPV).

Desde 2010, combina el trabajo en restauración con la investigación en la aplicación de mecanismos de conservación sobre arte público independiente y Urban Art. Al mismo tiempo, ha trabajado en diferentes proyectos de investigación en conservación de pintura mural, ha participado en conferencias y simposios internacionales en diferentes ciudades Europeas, y cuenta con publicaciones en español e inglés relativas al estudio, conservación y difusión del grafiti y el arte urbano y, desde 2014, ha colaborado activamente durante el proceso creativo con las artistas Patricia Gómez y Ma Jesús González, además de hacer entrevistas a artistas vinculados al grafiti y arte urbano, como parte de su investigación. Es miembro del grupo de trabajo de Arte Urbano del GE-IIC por la defensa de buenas prácticas en la preservación de arte urbano.

En los últimos años, ha trabajado en programas educativos y de difusión del patrimonio británico dentro de instituciones públicas como la Royal Academy of Arts y los Royal Museums Greenwich. Actualmente trabaja en el sector privado en conservación, preservación y en la preparación de exposiciones a nivel internacional. 